\title{
La resistenza culturale nel Libano contemporaneo. Le sfide di artiste locali e profughe
}

Cultural resistance in contemporary Lebanon: The challenges of local and refugee artists

\section{Estella Carpi e Stefano Fogliata}

\section{OpenEdition}

\section{Journals}

Edizione digitale

URL: http://journals.openedition.org/aam/3502

DOI: $10.4000 /$ aam.3502

ISSN: 2038-3215

\section{Editore}

Dipartimento Culture e Società - Università di Palermo

Notizia bibliografica digitale

Estella Carpi e Stefano Fogliata, «La resistenza culturale nel Libano contemporaneo. Le sfide di artiste locali e profughe», Archivio antropologico mediterraneo [Online], Anno XXIII, n. 22 (2) | 2020, online dal 31 décembre 2020, consultato il 28 janvier 2021. URL: http://journals.openedition.org/aam/3502 ; DOI: https://doi.org/10.4000/aam.3502

Questo documento è stato generato automaticamente il 28 janvier 2021.

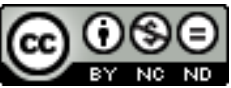

Archivio antropologico mediterraneo è distribuita con Licenza Creative Commons Attribuzione - Non commerciale - Non opere derivate 4.0 Internazionale. 


\title{
La resistenza culturale nel Libano contemporaneo. Le sfide di artiste locali e profughe
}

\author{
Cultural resistance in contemporary Lebanon: The challenges of local and \\ refugee artists
}

Estella Carpi e Stefano Fogliata

\section{Introduzione ${ }^{1}$}

1 Nella regione nordafricana e mediorientale, la storia politica non è soltanto quella di liberazione da uno Stato autoritario, e il Libano - in cui lo Stato classicamente inteso come apparato burocratico centralizzato e sovrano in materia di sicurezza interna - è sempre stato opportunisticamente assente, ne è un esempio lampante. Nella storia contemporanea del Libano, numerosi sono stati gli sforzi dal basso di resistere al potere di uno Stato confessionalista - ovvero organizzato per seggi assegnati rispetto all'appartenenza comunitaria (ta'ifa), secondo il quale il Presidente della Repubblica deve essere Cristiano maronita; il Primo Ministro musulmano sunnita; il portavoce del Parlamento musulmano sciita. In tale scenario politico, in cui si oppongono varie forze e visioni non solo legate alla ta'ifa ma soprattutto alle complessità regionali e sociali di larga scala, la "resistenza" non emerge come processo fisso e lineare di opposizione. In tale scenario, la figura del migrante (soprattutto se forzato, vittima di conflitti, carestie o insostenibili condizioni economiche) diventa strumento di politica nazionale e, nel caso libanese, pedina fondamentale per il mantenimento dell'equilibrio demograficoconfessionale. In tale articolo, non proponiamo una nostra propria concettualizzazione di "resistenza culturale", ma adottiamo invece il concetto quale proposto - in modo diversificato - da ognuna delle artiste intervistate. In modo analogo, gli autori hanno lo scopo di far emergere tali molteplici interpretazioni di "resistenza" vis-à-vis l'entità dello Stato, il quale, a dispetto di una vasta letteratura che tende ad articolarne la definizione (Das, Poole 2004; Reno 2011; Mouawad, Baumann 2017), spesso incarna, dal 
punto di vista degli attivisti, un'entità relativamente omogenea, opprimente e inaffidabile.

2 Resta quindi complesso identificare chi governa de facto e chi oppone resistenza con vari mezzi per sopravvivere, per proliferare, o per prevaricare. Laddove la cultura non può essere definita solo in termini comunitari, il campo politico e quello culturale si incontrano, si schivano e si intrecciano in maniera alquanto complessa. In modo analogo, i cambiamenti che avvengono nell'arena culturale non fungono necessariamente da barometro di cambiamenti economici e politici più ampi, ma talvolta restano di dimensione limitata, specialmente in paesi con ministeri della cultura centralizzati e forti come l'Egitto, la Siria e la Tunisia (Pahwa, Winegar 2012). Negli Stati-nazione, l'ideologia istituzionale dominante posiziona i segmenti di cultura "resistente" come percorso umano verso il progresso intellettuale, artistico o sportivo. Pertanto, laddove i ministri della cultura tendono a voler controllare e plasmare l'ambito culturale, si tende a pensare che lo Stato si ponga esclusivamente come impedimento alla libertà d'espressione e alla proliferazione di forme indipendenti di cultura.

3 Il Libano, uno Stato storicamente alla mercé di poteri esteri - quali gli Stati Uniti d'America, l'Arabia Saudita, l'Iran e la Francia in primis - a maggior ragione, non presenta una semplice dicotomia tra società che aspira a una cultura libera e uno Stato oppressivo. Se la cultura è spesso inibita, violentata o limitata a causa di azioni provenienti dalle istituzioni statali, è anche lo Stato libanese stesso che, in tempi di crisi umanitaria, spesso non è intenzionato a serrare la porta ad approcci neoliberisti e investimenti provenienti dall'esterno (Mac Ginty 2007; Baumann 2014). E se questi ultimi da un lato stanno di fatto finanziando la produzione culturale all'interno dello Stato, dall'altro stanno indebolendo l'originale intento politico e trasformativo di tale produzione culturale, rendendola pura scelta estetica di alcune classi sociali e gruppi diasporici e riducendola, dunque, al rango di stile di vita transnazionale.

4 È sulla concettualizzazione endemica di resistenza culturale e il modo in cui gli artisti la esercitano che vuole focalizzarsi il presente articolo, partendo da interviste condotte in lingua araba e inglese dai due autori nel 2018 con due politologi libanesi, un'artista libanese, una giornalista specialista in produzione artistica contemporanea in Libano, un'artista siriana e due artisti palestinesi in Libano. Le artiste selezionate per le interviste non solo sono figure conosciute in Libano, ma sono anche rappresentative delle diverse componenti demografiche e sociali del Paese. Questo articolo si colloca anche alla fine di un lungo percorso di lavoro etnografico che ognuno dei due autori ha eseguito nel corso degli ultimi anni in Libano con migranti e profughi provenienti da diverse parti della regione mediorientale. Gli esponenti chiave della scena culturale e artistica che abbiamo intervistato non sono quindi solo cittadini libanesi, ma spesso rifugiati o migranti, che hanno cercato di ritagliarsi un posto nella società libanese per permettersi di vivere d'arte e di cultura. Le interviste con entrambi i gruppi sociali ci hanno inoltre permesso di identificare le aree comuni di potenziale incontro.

5 Abbiamo scelto di concentrarci sul concetto e sulle modalità di resistenza nell'ordinarietà quotidiana esplicate attraverso l'arte e la produzione culturale ampiamente intese. Se nel mondo arabo si parla usualmente di produzione culturale come plasmata dal modo in cui la produzione culturale e la storia nazionale si intrecciano nelle lotte contro il colonialismo, l'imperialismo e il capitalismo (Winegar 2006: 9), il Libano si colloca in quest'arena in modo complesso. Qui il lavoro culturale 
inevitabilmente mette in luce l'adattamento di diverse generazioni alle trasformazioni globali e regionali. Questo ricorda il concetto di "generazione" proposto da Winegar (2006), la quale, analizzando la scena culturale egiziana nel suo proprio contesto politico, definisce "generazionale" il modo in cui gli artisti egiziani spiegano l'organizzazione e il cambiamento sociali sulla base di cui lavorano gli artisti della stessa o diversa età. In questo tentativo di dissotterrare la linea che corre attraverso la produzione culturale e i cambiamenti politici in Libano, intendiamo muovere l'interesse dei lettori ben oltre la mera valutazione etica di un Libano in cui la resistenza culturale possa essere o un "copia e incolla" dai paesi occidentali, o soltanto un veicolo di autoctonia culturale. Tanti sono stati i tentativi di collocare i paesi mediorientali - e, più in generale, i paesi del cosiddetto Sud globale - lungo un ingannevole rettilineo che parte dalla tradizione e arriva alla modernità e al neoliberismo culturali. Questi tentativi hanno invece perso l'occasione di soffermarsi sulle peculiarità contestuali di ognuno di questi paesi, e della loro diversificazione interna in termini di resistenza e cultura.

Quando parliamo di "resistenza culturale" ci riferiamo alla presenza di attività e pratiche culturali all'interno di un contesto ostile e in cui è arduo farsi spazio. Come ha sottolineato Nathalie Rosa Bucher, giornalista esperta di arte e produzione culturale in Libano (intervista del 28 maggio 2018):

Le sfide possono essere la mancanza di risorse, il supporto istituzionale che pone delle condizioni vincolanti, la censura o comunque ristrettezze legislative, e la mancanza di spazi per mostrare o attuare il lavoro artistico. Per me la resistenza culturale è quindi l'abilità di sapere mantenere certe tradizioni artistiche, ma anche reinterpretare e modificare queste ultime in nome di una continuità resiliente.

7 Siamo innanzitutto interessati a comprendere che cosa significhi il concetto di resistenza culturale per i "fautori della cultura" - come, d'ora innanzi, chiameremo sommariamente artisti propriamente detti e produttori di qualche forma di cultura, per riferirci sia al loro mestiere professionale che al loro contribuito alla vita culturale libanese e regionale - siano essi cittadini o migranti nel Libano contemporaneo. Cercando di comprendere come la scena artistica si sia evoluta negli anni successivi alla fine della guerra civile libanese (1975-1990), siamo particolarmente interessati a capire come essa si sia evoluta in seguito all'arrivo dei flussi di rifugiati dalla regione, e dopo l'arrivo massiccio dell'industria umanitaria a seguito delle crisi politiche nel Levante arabo negli ultimi due decenni - in primis Iraq e Siria. Focalizzandoci principalmente sul fenomeno dei migranti, che costituiscono ormai una parte integrante del tessuto sociale e culturale libanese, intendiamo esaminare come si configuri lo spazio per l'artista "straniero" - qualsiasi sia il suo status legale - all'interno della società libanese. In questo senso, questo articolo affronta principalmente la politica della cultura - sede di lotte politiche e ideologiche (Mahoon 2000: 470 in Winegar 2006) che nasce in primis nella politica propriamente detta.

8 La scena artistico-culturale in Libano è articolata sul livello sia generazionale che politico (Haugbolle 2012), il quale riflette gli scismi ideologici della società locale. Un irrisolto crogiuolo di memorie della guerra civile, a seguito di un'amnistia generale che evitò di condannare i leader di guerra dopo il conflitto civile, è spesso utilizzato da artisti e intellettuali per spianare la strada a sentimenti nazionali, seppur non necessariamente nazionalisti. Il lavoro artistico e culturale emerge quindi come uno script pubblico che si esprime ideologicamente su questioni politiche e sociali; tuttavia, 
come evidenziato dai nostri intervistati, il diritto degli artisti a esprimersi su tali questioni è stato talvolta contestato.

9 La crisi siriana del 2011 ha costituito un importante spartiacque nella scena artistica e culturale libanese, legando i finanziamenti di eventi culturali e di mostre alla crisi di emergenza. Questo rende estremamente vulnerabili le attività finanziate in tal modo, poiché la loro temporalità resta ineluttabilmente legata a quella in cui si svolge l'emergenza che, come noto, perde di priorità finanziaria e mediatica con l'andare del tempo. Questo fenomeno, tuttavia, ha permesso alle crisi umanitarie e politiche di più vecchia data - come quella palestinese - di riottenere interesse pubblico e attenzione internazionale. A questo proposito, per la studiosa di politica economica libanese Lea Bou Khater (13 giugno 2018), lo sviluppo, le crisi e l'arte ormai procedono parallelamente: «Prima della crisi in Libano al limite avevamo solo le musalsalat (soapopera) siriane in termini di produzione culturale», ha affermato Bou Khater.

Le questioni odierne irrisolte del Libano contemporaneo, come dimostrano anche le attuali proteste della cosiddetta "rivoluzione d'ottobre" 2019 (thawrat October) e la precedente Garbage Crisis (crisi dei rifiuti) dell'agosto 2015, includono i diritti LGBT, la corruzione nella gestione dei rifiuti, la mancanza dei diritti sul lavoro e il crescente clientelismo sulla base dell'appartenenza confessionale, che viene ora potentemente sfidato dalle mobilitazioni popolari dopo esser riemerso in modo preoccupante nella vicina Siria devastata dal conflitto. I fondi investiti nella produzione culturale sul suolo libanese sono maggiormente orientati verso iniziative umanitarie, al fine di gestire "traumi" post-guerra, e, pertanto, di "medicalizzare" delle questioni di natura prettamente politica. La tendenza a "patologizzare" questioni che necessitano una risoluzione politica, ampiamente discussa da studiosi internazionali (Das et al. 2001; Fassin, Rechtman 2009), ha spesso imposto al soggetto, attore e vittima di processi politici, di sottoporsi a programmi internazionali al fine di ricevere una "diagnosi" e venir "guarito". In questo scenario, i fautori della cultura, sia migranti sia cittadini locali, cercano di orientarsi verso le questioni di migrazione forzata, di genere, di infanzia e gioco e, in misura minore, di ambiente e miglioramento dello spazio urbano.

11 Nonostante il Libano vanti una lunga vita culturale e artistica, in base alla nostra analisi delle forme culturali odierne attraverso cui si sviluppano forme di resistenza locale, emerge una domanda: l'autonomia artistica e culturale dalle istituzioni statali è illusoria? La storia araba stessa ha talvolta dimostrato che sono le agende riformiste di Stato a dare vita agli spazi culturali (Pahwa, Winegar 2012). Per esempio, durante gli anni '50 e ' 60 , la produzione culturale egiziana faceva parte del processo di state-crafting (come analizzato ampiamente da Winegar 2006). Il fatto che il Libano possieda delle strutture statali considerate deboli o inaffidabili non risolve il quesito, spostando il focus dalle istituzioni statali a quelle non statali, che tuttavia incarnano un potere simile a quello di Stato. In questo genere di scenario, l'arte può portare al cambiamento? Le visioni culturali, gli artisti e in generale i fautori della cultura possono essere sottomessi al potere politico, ma possono anche perpetuarlo e riprodurlo. Nel caso del Libano, dimostreremo come l'impasse che deve affrontare la cultura, qui intesa come efficace strumento di resistenza e cambiamento, sia dovuta all'esteticizzazione e alla conseguente anestetizzazione delle forme di resistenza, svuotate del loro potenziale politico, e prevalentemente finanziate in qualità di pacchetto umanitario e di stabilità sociale. 


\section{II contesto storico-politico}

12 «Talvolta la vita culturale segnala decadenza, invece che resistenza». Hisham Safieddine, studioso di politica libanese presso il King's College di Londra, esordisce così alla nostra richiesta di parlare di che cosa sia la resistenza culturale in Libano oggigiorno (12 luglio 2018). A questo proposito, Safieddine, ci offre un quadro completo della storia politica libanese degli ultimi venti anni, dimostrando come alcune correnti culturali non emergono necessariamente in opposizione a una forza che le reprime, e quindi non necessariamente come veicolo di "resistenza".

13 Il clima politico degli anni della guerra civile libanese (1975-1990) non aveva permesso alla società civile di fiorire da un punto di vista culturale: «Il Libano era ed è ancora dominato da élites, poteri locali informali, oltre che dal regime siriano durante la cosiddetta Pax Syriana (1975-2005)». L'esplosione urbana e il fiorire del settore privato interessano gli anni immediatamente successivi alla fine della guerra civile, chiusa dall'accordo di Ta'ef (1989-1990). Gli ambienti della sinistra politica libanese hanno finito per abbracciare il neoliberismo promosso dal leader del partito al-Mustaqbal - "Il futuro" - Rafiq al-Hariri, attraverso società come la sua Solidère (fondata negli anni ' 90 per ricostruire il centro di Beirut dopo la guerra civile), per riuscire a superare l'effetto debilitante della guerra (Baumann 2014). Nel decennio 1990-2000 i servizi sia di base che terziari del paese finiscono in mano ad investimenti esteri (Ibidem). È in questo periodo che i mass media, e parte della produzione culturale locale, creano una connessione stretta con i partiti politici libanesi.

14 Come afferma Safieddine, i modi in cui la resistenza viene intesa a livello nazionale in Libano sono diversi e, a loro volta, generano diversi concetti di resistenza nella società stessa:

La resistenza in Libano può essere quella del partito [maggiore sciita] di Hezbollah a Sud e nella valle della Beqaa, e in questo caso ha portato a una liberazione dall'occupante israeliano nel maggio 2000. Per noi libanesi anche il 2003 è stato un anno fondamentale, poichè gli Stati Uniti d'America hanno voluto ridisegnare il proprio ordine politico in Medio Oriente tramite l'invasione dell'Iraq.

15 Analogamente, il ritiro delle truppe dell'esercito siriano di Bashar al-Asad nel 2005 era stato incoraggiato dalle politiche di stati occidentali, oltre che dalle proteste libanesi in piazza del 14 marzo 2005 (in seguito battezzate la Rivoluzione dei Cedri), avvenute a seguito dell'assassinio dell'ex premier Hariri nel febbraio 2005. La resistenza a livello interno libanese si configura anche come opposizione a, o in supporto di, cambiamenti regionali, i quali hanno causato indirettamente cicliche crisi di stabilità.

16 Il settarismo libanese, ampiamente discusso nella letteratura internazionale (Makdisi 2000), gioca un ruolo fondamentale nell'ampliare ulteriormente il concetto di resistenza culturale nel contesto libanese. Nell'intervista condotta, Safieddine evidenzia l'importanza del neoliberismo e della privatizzazione del settore terziario postguerra - il cosiddetto harirismo - come tassello fondamentale nel rafforzamento delle linee di separazione tra le identità settarie libanesi: «Sono questi gli anni in cui possiamo parlare di mazlumiyye ("oppressione") più da un punto di vista confessionale che politico. Di riflesso, rispecchiando le identità confessionali sunnite e sciite rispettivamente, l'harirismo in Libano rappresenta ancor oggi la politica saudita, e l'hezbollismo la politica iraniana in Libano». 
17 Nella recente storia politica libanese, emergono quindi i seguenti punti di consenso e dissenso culturali: in primo luogo, l'harirismo promuove il neoliberismo, che influisce sulla cultura consumistica odierna nel paese ma allo stesso tempo non trova i fautori della cultura dalla sua parte; e, in secondo luogo, la posizione socio-politica dei fautori della cultura rispetto alla questione israeliana, i quali approcciano la resistenza in modo diversificato. Safieddine specifica come, nel postguerra, abbia avuto luogo un'identificazione di alcune correnti culturali libanesi nella figura di Hariri, ma sottolinea che alcuni tratti di una politica culturale alquanto variegata sono in realtà il prodotto di quindici anni di guerra civile libanese:

I libanesi dopo la guerra bramavano uno spazio nazionale per la cultura e per l'espressione di pratiche quotidiane di resistenza. È così che le correnti intellettuali hanno ricominciato a proliferare, soprattutto nell'ambito di piccole università private nella capitale Beirut.

In particolare, dopo l'assassinio di Hariri per mano indiretta del regime siriano questione tuttora disputata da un Tribunale Speciale per il Libano (Humphrey 2011) - si apre uno spazio di possibilità, dove altri poteri hanno la potenzialità di emergere in un clima generale di incertezza.

La storia geopolitica libanese, dopo il ritiro di Israele dal Sud (maggio 2000) e della Siria dall'interno del paese (aprile 2005), è un susseguirsi di resistenze sviluppate su più livelli e reagendo a più oppressori. Prima della guerra di luglio 2006 tra Israele e il Libano, il partito libanese Hezbollah - primo target degli attacchi israeliani - e il regime siriano erano tutt'altro che coesi. Dopo la guerra, una opinione pubblica più omogeneamente consenziente nei confronti di Hezbollah e della sua vittoria pirrica su Israele - che costò la vita a più di un migliaio di persone - sembrava emergere. Secondo Safieddine, questa tendenza politica, dopo gli importanti eventi del 2005 e 2006, si intreccia a una ghettizzazione della vita culturale lungo linee settarie e confessionali che, negli ultimi anni, a seguito della guerra siriana (2011-), emergono in modo più marcato. Inoltre, a marcare ulteriormente le linee tra i gruppi, risorge un clima di mutuo sospetto. Le attuali proteste libanesi di massa, iniziate il 17 ottobre 2019, sembrano però suggerire una contestazione popolare di vasta scala contro tale conformazione sociale frammentata, a cui fino ad ora non c'è stata risposta politica.

20 È in questo senso che Safieddine definisce la cultura «una politica dell'ultima spiaggia». Ovvero, in assenza di azione politica, la produzione culturale non può certamente rimpiazzare la prima, ma può diventare una forma supplente di resistenza, seppur fragile. È fragile perché dà solo l'impressione di essere resistenza, ma è mera compensazione per la mancanza di atti politici che possano produrre un cambiamento. A veduta di Safieddine, in Libano c'è assenza di un progetto politico istituzionale, e questo produce anche un malessere culturale. Safieddine, che è stato fondatore della versione inglese del quotidiano libanese al-Akhbar - letteralmente, "le notizie" - pensa che i mezzi di informazione ed espressione possano essere un modo efficace per lavorare verso l'azione politica. Invece, la menzionata crisi dei rifiuti dell'estate 2015, che chiamò centinaia di persone in piazza a protestare senza portare a risultati effettivi, esemplifica come la produzione intellettuale abbia finora fallito a tradursi in un progetto politico collettivo, ma soltanto in tendenze individuali.

21 A tal proposito, Tania El Khoury, live artist libanese di fama internazionale, autrice di installazioni artistiche, e ricercatrice interessata al potenziale politico del lavoro artistico, rimarca quanto la sua produzione e ricerca sia più orientata a stimolare una 
cultura di resistenza piuttosto che una resistenza ch'ella definisce "acculturata" (12 settembre 2018):

La cultura di resistenza è una cultura in cui ogni membro della comunità - artista o meno - usa il proprio lavoro per sfidare la centralizzazione del potere. La resistenza acculturata rischia invece di far dell'arte e della cultura delle ancore di salvezza della società: una posizione di cui sono diventata critica. Nel contesto libanese c'è certamente un'intersezione tra gli artisti, chi lavora all'interno del settore culturale, e gli attivisti: siamo tutti coinvolti in questioni legate all'eguaglianza di genere, la giustizia sociale, la giustizia spaziale e la rappresentatività nella politica locale. Abbiamo visto rappers e illustratori essere a capo delle proteste che contrastavano la garbage crisis del 2015. Abbiamo visto accademici e produttori cinematografici competere nelle elezioni municipali. Abbiamo visto campagne per la conservazione di spazi ed edifici, a rischio di smantellamento a causa delle politiche governative, per cui si battevano artisti e ricercatori. Nonostante uno sforzo significativo e un ampio supporto popolare, queste persone sono ancora una sparuta minoranza nella società libanese e restiamo quindi lontani un miglio dal poter creare una cultura di resistenza. Possiamo comunque dire che il settore culturale indipendente in Libano si stia adoperando per sviluppare varie strategie di resistenza.

La grossa presenza di aiuti internazionali nella società libanese in risposta alle numerose crisi umanitarie di lunga durata è essa stessa una forma di resistenza culturale che edulcora la mancanza di tangibili alternative politiche e istituzionali. Se la società civile libanese lotta per spazi più ampi di espressione, i meccanismi attraverso cui funzionano questi movimenti civili restano prevalentemente non-politici o, in ogni caso, ufficiosamente politici. Spesso, i finanziamenti stessi per l'ottenimento di tali spazi vengono assegnati a condizione che questi ultimi non si configurino come politici. «La neutralità è di moda, i partiti politici libanesi utilizzano oggi prevalentemente un linguaggio ammorbidito per questa ragione. La politica odierna si muove per lo più in tempo di elezioni e per creare coalizioni effimere», afferma Safieddine.

Questo percorso sul piano politico ed ideologico ha avuto un impatto nettamente negativo sulla produzione culturale e, secondo Safieddine, anche sulla stampa: «Non vi è più stampa affidabile, c'è solo propaganda. Una certa ideologia sociale libanese può essere progressista ma ciò è riflesso nella stampa in misura sempre minore, e questo è alquanto preoccupante, perché non si fa più dell'informazione propriamente detta». Seppur si possa difficilmente trovare un paese in cui l'informazione non sia per nulla politicizzata, come emergerà nelle interviste condotte con le artiste, gli spazi di discussione devono essere gelosamente custoditi e sviluppati per mantenere un margine d'azione. A questo proposito, Safieddine menziona i magazine online come al-arabi al-jadeed ("Il nuovo arabo") che hanno ottenuto un vasto seguito, ma ritiene che essi si configurino più in forma di iniziative che di vere e proprie presenze politiche. È dunque in un paesaggio mediatico impoverito che il consumismo libanese di beni e servizi di lusso, frutto dell'harirismo politico postguerra (Khalaf 2006), e la corrente neutralizzazione del fattore politico tendono a fagocitare iniziative artistiche e culturali nel Libano contemporaneo: questo fenomeno tende a mercificare la cultura e a presentarla in forma di terapia psicologica, rendendo le lotte politiche interne e migranti delle mere risposte alle crisi umanitarie. 


\section{La resistenza culturale nel Libano contemporaneo}

'For us art is resistance: The resistance that makes ourselves unforgotten.

Art is a sort of signature that we are here,

and you cannot deny our own existence'

(Rasha Salah, palestinese di Libano, Dar an-Nimer, 13 giugno 2018).

Nell'ultimo decennio, forme di espressione culturale hanno guadagnato terreno anche fuori dalla capitale Beirut. Beirut resta tuttavia il cuore centrale della maggior parte delle attività culturali in Libano. Nel 2013, è sorto per esempio il MACAM, Modern and Contemporary Art Museum, che si trova nella località di Alita; come anche Hamama Artist House, fondata a Hamama nel 2017. Anche il numero dei festival è considerevolmente aumentato. Mentre negli scorsi decenni i festival venivano per tradizione organizzati principalmente a Baalbek (a partire dal 1957), ora queste manifestazioni di respiro nazionale hanno luogo anche a Beiteddine, Byblos, Ehden, Batroun e Jounieh. Anche municipalità più piccole come Ghalboun cercano di attrarre un grosso numero di spettatori, come nel 2017 Ehdeniyat, dove ha avuto luogo un concerto della popolare pop-band libanese Mashrou' Leyla. Anche il numero delle gallerie d'arte è aumentato vertiginosamente. Per esempio, a Beirut, Saleh Barakat Gallery, nel distretto di Clemenceau, è stata costruita all'interno di un vecchio cinema; Galerie Tanit si trova vicino all'Electricité du Liban nel quartiere di Mar Mikhael an-Nahr; Sfeir-Semler a Qarantina; Artlab a Jemmayzeh; e Janine Rubeiz Gallery a Raouche.

Nonostante ciò, alcuni siti ritenuti fondamentali nella produzione culturale locale sono stati chiusi, come il Masrah Beirut a 'Ayn al-Mreisse, che è stato storicamente un punto di incontro sociale e culturale di grande importanza nella movida beirutina. Il progetto Beit Beirut, che sarebbe dovuto diventare un museo della memoria nella città di Beirut, è stato completato senza che venisse riconosciuto come istituzione culturale. Tale progetto artistico è il primo di natura pubblica all'interno della municipalità di Beirut, ma non c'è stata infine la volontà politica di rendere tale spazio qualcosa che andasse oltre l'organizzazione di mostre ed eventi: manca difatti un'istituzione che includa spazi e risorse per fare ricerca, archivi e biblioteche. Tuttavia, la maggior parte degli eventi avviene in spazi privati a Beirut. Negli spazi pubblici si contano il Cabriolet Film Festival sulle scale di San Nicolas, e La Fête de la Musique. Inoltre, la riapertura del Sursock Museum dopo sette anni dai lavori di ristrutturazione ha rinverdito la vita culturale beirutina. L'accesso alla maggior parte degli eventi è gratuito. Eventi di iniziativa privata proliferano, come Mansion a Zoqaq el-Blatt che è stato edificato in una vecchia residenza privata con il consenso del proprietario, ed è ora diventato un punto di incontro per artisti e attivisti. In modo simile, Beit Waraq a Noueiri, che prima era un open space, è diventata un luogo per collettivi che si occupano di design, organizzano workshops, e fanno animazione. Badguer nel quartiere armeno di Beirut, Bourj Hammoud, celebra la creazione di manufatti locali e la cultura armena: ospita anche un ristorante e organizza eventi al suo interno. Di recente fondazione, nel 2016, è anche la Dar an-Nimer, che promuove l'arte e la cultura palestinesi, allargandosi anche al mondo arabo in generale. Situata nel cuore di Clemenceau a Beirut, la Dar an-Nimer ospita eventi artistici, proiezioni cinematografiche, eventi musicali, presentazioni di libri, dibattiti, e laboratori di vario genere. Si cimenta anche nell'organizzazione di eventi all'interno dei campi palestinesi, diventando un importante nodo connettore tra l'arte araba, quella libanese e quella palestinese. Un'organizzazione non-governativa che funge da importante connettore attraverso l'intero mondo arabo, spesso occupandosi 
di conflitto, violenza e solidarietà al di là dei confini nazionali, è la Arab Puppet Theatre Foundation (APTF) con sede a Hamra, quartiere di Beirut che ha storicamente costituito il centro della capitale, seppur localizzato nella parte occidentale della città. La pratica delle marionette può vantare un ampio patrimonio all'interno del mondo arabo: l'APTF ha rispolverato una lunga tradizione, ancora troppo sconosciuta su un piano internazionale, per fare dell'arte uno strumento pedagogico adatto a tutte le età.

Alcuni generi artistici degli inizi del ventesimo secolo, come la pittura paesaggistica libanese di cui parla la studiosa Kirsten Scheid, si son fatti portatori di determinati pensieri politici, quali un'etica di cittadinanza non settaria (Scheid 2015: 349). La maggior parte dei fondi che sostengono tali iniziative proviene da ambasciate straniere e istituzioni finanziate dall'esterno. A dispetto di ciò, l'impatto di questi eventi ha un ruolo fondamentale nella trasformazione della società libanese odierna. Tali spazi hanno attratto audience diverse da varie aree residenziali, e ne hanno ridefinito le identità. Il fatto che l'entrata agli eventi e alle mostre sia spesso e volentieri gratuita ha ulteriormente ampliato - e pertanto diversificato - l'audience.

Secondo la maggior parte dei nostri intervistati, gestire le relazioni con le politiche libanesi per produrre arte, sport e cultura è molto arduo. Come ha affermato Dima Nashawi (11 maggio 2018), artista siriana specializzata in disegno grafico e calligrafico, profuga di guerra, e ora residente a Beirut:

Il governo è spesso passivo e pone parecchi ostacoli, anche se non c'è una vera e propria policy sulla produzione culturale in Libano. Inoltre, anche quando ci si candida a un festival, è dura essere selezionate se si presentano lavori in qualche modo esplicitamente politici. Questa è cecità, giacchè è proprio il movimento artistico civile ad aver incrementato la comunicazione tra le varie parti confessionali nel Levante arabo. Gli artisti hanno aperto gli occhi anche sulle questioni di genere in Libano. È per questo che il ruolo dell'artista per me non dovrebbe essere assolutamente elitista. In questo la rivoluzione siriana e la crisi che è seguita hanno aiutato parecchio a rendere l'arte uno strumento di trasformazione sociale.

Perdipiù, è fondamentale ricordare che l'arte in Libano sia storicamente connessa a finanziamenti inglesi e francesi. A questo proposito, gli artisti più finanziati sono coloro che sono in diverse forme connessi a reti internazionali e spesso multilingue. Come ha testimoniato Dima Nashawi (11 maggio 2018), «Se sei fuori da questi circoli è difficile lavorare, ma la scena artistica e culturale ultimamente è comunque diventata più liberale a causa dei flussi migratori verso il Libano. Questi hanno aumentato l'interesse verso l'arte prodotta da rifugiati siriani come me, e si è creato un piccolo spazio anche per noi, fornendoci più risorse». A tal proposito, nell'intervista condotta, l'artista libanese Tania El Khoury ribadisce (12 settembre, 2018):

Pochi anni dopo che le proteste popolari in Siria esplosero nel 2011, la scena culturale di Beirut appariva molto vivace. A mio avviso, l'arrivo in Libano di artisti, intellettuali e attivisti siriani ha segnato la città in modo positivo. Per coloro che lavoravano a Beirut durante quel periodo, era facile incontrare e collaborare con i nuovi arrivati siriani. Il loro e il nostro lavoro si sono trasformati parecchio. La scena urbana vivace e indipendente dell'arte contemporanea lascia certamente un segno sui nuovi arrivati. Aggiungerei anche che la sollevazione siriana, così come l'incontro con gli artisti e attivisti siriani, hanno influenzato il lavoro di molti artisti libanesi, me inclusa.

Con il recente arrivo delle agenzie umanitarie in Libano, che si propongono di assistere i profughi dalla Siria, il lavoro artistico è stato commissionato in misura crescente sotto 
forma di impegno sociale invece che di strumento in mano ai circoli elitari. Ciò è significativo soprattutto in un paese in cui l'arte ha a lungo riflesso le preferenze e tendenze culturali delle classi sociali più alte. Facciamo l'esempio della membership card ai circoli artistici che circolava negli anni '30 a Beirut, scritta in francese negli anni del mandato, e raffigurante immagini greco-romane, levantine e beirutine (Scheid 2009: 112), utilizzata da élites che avevano sviluppato uno stretto rapporto con $\mathrm{i}$ rappresentanti locali del mandato francese in Libano (1920-1946). Oggigiorno, invece, vari artisti in città e campi profughi sono stati coinvolti per lavorare con bambini e giovani, a cui si destinano vari progetti umanitari. Pertanto, la presenza dei flussi migratori - e una risposta umanitaria senza precedenti, che tali flussi hanno attratto nel Libano attuale - si pongono sia come un limite che come un'opportunità rispetto al lavoro culturale. A questo proposito, Nashawi afferma:

Il problema è che quando i fondi sono forniti da ONG legati alle crisi, i progetti sono temporanei. Quando emerge un'altra crisi umanitaria, il più delle volte il progetto resta incompleto. Questo ha un impatto negativo non solo su noi rifugiati, ma anche sui cittadini libanesi in qualità di artisti.

30 A vedere delle artiste, l'aspetto positivo della commissione umanitaria è di far uscire ulteriormente l'arte e la cultura dall'elitismo. Come ha affermato Rasha Salah (13 giugno 2018), co-fondatrice e direttrice esecutiva della Dar an-Nimer e originaria di ‘Ayn al-Helwe:

Le mostre per noi sono solo una strategia, un modo per arrivare a più gente. A molti non interessa l'arte, perché non sono stati esposti all'opportunità di comprenderla e volerle lasciar spazio. È per questo che organizziamo laboratori che coinvolgano i bambini, è un modo di coinvolgere anche i loro genitori. Non vogliamo che l'arte sia elitaria, ma che dia la possibilità di dare spazi sicuri ai giovani palestinesi.

31 Il paradosso risiede nel fatto che gli artisti difficilmente concepiscono il proprio lavoro in senso umanitario, ma perlopiù politico. Come afferma Nashawi (11 maggio 2018), «Per noi artisti siriani l'arte nasce come collettività e per la nostra causa, la quale è necessariamente politica. Non c'è niente che si chiami arte al fine di essere soltanto... arte». Ad esempio, il lavoro artistico è maggiormente commissionato in clima elettorale, o in concomitanza con sollevazioni popolari: in Siria ad esempio è considerevolmente aumentato dopo le proteste del 2011, mentre in Libano l'arte sia elitaria che di strada era presente sin da prima, essendo un paese caratterizzato da numerosi tumulti popolari e instabilità politiche (Nashawi, 11 maggio 2018). La politica interna libanese e lo spazio di espressione relativamente liberale di cui gode il Libano han fatto sì che il paese offrisse mezzi e spazi maggiori per espressioni di resistenza culturale anche di altri popoli migranti all'interno del paese. Ad esempio, in Libano si ha accesso a più materiali, a più tecniche e a più fondi. «In Siria il regime di Asad soffocava queste possibilità», sottolinea Nashawi.

In questo senso, secondo Nashawi, la resistenza culturale e politica per i siriani in Libano si muove di pari passo con la scelta di essere artista. Con l'incremento della domanda artistica in Medio Oriente da parte del mercato internazionale, vi è dunque un incremento delle vie che si possono percorrere per "resistere". È per questo motivo che l'esperta Nathalie Rosa Bucher parla di una "seconda Nahda" culturale dopo le sommosse arabe del 2011.

Di fronte alla contraddizione di finanziamenti a fine umanitario da un lato e, dall'altro, cause prettamente politiche che muovono la scena artistico-culturale, la neutralità politica viene descritta dai fautori della cultura come un danno morale. Come ha 
affermato il musicista siriano Mohammad Abu Hajar (2019) in merito alla canzone Libiladi al-mutanna, "Ai nostri paesi", interpretata da Faya e Rihan Younan:

Neutrality is a victory for those who have power at a specific moment, and thus the problem of the neutral song becomes that when it claims not to take a political stance and prefers to adhere to taking humanistic positions, it is trying to outsmart history, the public and perhaps the party who produced or the artwork in an attempt to delude everyone that it has no stance. All this while standing to the right in all social conflicts. Art, even if it doesn't confront, cannot lament, otherwise critics will cast it as defense of the old worn-out City in the face of attempts for renewal.

L'esempio di Dar an-Nimer dimostra ulteriormente come l'arte e la cultura vengano percepite come forme di attivismo tout court dai fautori stessi, malgrado le intenzioni e le politiche di chi le finanzia. Nella Dar an-Nimer, fondata da Rami an-Nimer collezionista d'arte, banchiere in origine - vi è un'importante collezione di fotografie e testi sulla storia della Palestina. Il fare cultura è qui concepito come un modo di continuare a lottare per il diritto al ritorno - haqq al-'awdeh - verso le terre occupate da Israele a partire dal 1948, anno della "catastrofe" palestinese (la Nakba), che segna l'esodo. Il lavoro svolto in questo spazio si pone come fine secondario quello di connettersi con altri rifugiati palestinesi nella regione, soprattutto con coloro che risiedono ad Amman. Analogamente, i profughi palestinesi di Giordania tentano di costruire uno spazio di comunicazione con la società libanese, con la quale avvenne una rottura soprattutto nel 1982, quando l'Organizzazione per Liberazione della Palestina (OLP) venne evacuata dal Libano. Come ha affermato Rasha Salah nell'intervista condotta (13 giugno 2018),

Manchiamo di uno spazio dove incontrarci e interagire, ma viviamo all'interno dello stesso paese. E penso che anche i libanesi stessi necessitino riconnettersi con la causa palestinese. È importante quindi che la nostra arte rompa gli stereotipi sui palestinesi in Libano... siamo descritti come gente senza arte né cultura. Abbiamo giocato un ruolo fondamentale nella guerra libanese, e ciò ha lasciato un'idea molto negativa dei palestinesi qui. Il nostro fine è dunque metter in dubbio quello che credono di sapere già... Insomma, l'arte non ha mai come fine l'arte stessa.

\section{Le sfide affrontate dai "fautori della cultura" in Libano}

L'alto costo degli affitti a Beirut è uno dei principali ostacoli che i fautori della cultura devono affrontare per potersi permettere uno spazio di creazione ed esibizione. Questo risulta essere un limite materiale soprattutto per gli artisti. La domanda di lavoro artistico sul mercato è comunque maggiore nella capitale, e i fautori della cultura raramente si spostano altrove: fuori da Beirut, si potrebbero forse permettere l'affitto, ma non avrebbero un numero sufficiente di commissioni. Le ristrettezze materiali, finanziarie e di spazio riguardano tutti gli artisti, sia cittadini libanesi che migranti e profughi. Al contrario, molte di queste limitazioni non riguardano invece gli artisti europei sempre più numerosi che transitano per Beirut. Tania El Khoury si esprime al riguardo:

Gli artisti occidentali che visitano il Libano o risiedono qui beneficiano di un certo numero di privilegi: dall'entrata nel paese senza visto, alla libera circolazione, e dalla non censura dei contenuti fino all'accesso a spazi altrimenti chiusi per gli artisti e i locali. Non importa quanto l'arte sia mediocre, una produzione artistica straniera (occidentale) in Libano è considerata arte rilevante che viene facilmente 
protetta e sponsorizzata, mentre molti artisti locali lottano per l'accesso agli spazi e ai fondi. siriani e iracheni in Libano devono ad esempio adattare il proprio stile al paese in cui vivono, al fine di assicurarsi la clientela. Per esempio, a questo proposito, Dima Nashawi afferma (24 maggio 2018): «Il khatt (segno) che utilizzo piace a seconda del contesto culturale di provenienza di chi lo commissiona, e a volte voglio del tempo per capire le preferenze dei clienti». A ciò si aggiungono i problemi legali dei cittadini siriani in Libano, a cui non è permesso aprire conti in banca. Gran parte degli artisti siriani riesce tuttavia ad accettare proposte di lavoro e commissioni escogitando modi per ricevere i pagamenti e offrire garanzie alla clientela, la quale tende normalmente a preferire conti in banca ufficiali. Come afferma Nashawi (24 maggio 2018):

Trovare modi per ricevere i pagamenti, garantire continuità lavorativa e sviluppare rapporti di fiducia con la clientela fanno parte della nostra resistenza culturale. In questo riusciamo molto bene, perché al momento gli artisti libanesi non hanno più lavoro di noi artisti siriani qui in Libano.

Difatti, altri artisti intervistati come Rasha Salah della Dar an-Nimer, hanno poi confermato che i libanesi stessi hanno iniziato a far fronte a una seria competizione in campo artistico soprattutto a seguito della crisi siriana, poiché i profughi stessi riescono ad attrarre maggiori fondi in qualità di artisti. Tuttavia, il capitale estero si indirizza principalmente verso la crisi e i suoi attori, e crea dunque una gerarchia tra gli artisti in Libano. Il problema in sé non è quindi di dover affrontare la competizione locale. Quest'ultima viene anzi guardata in modo positivo da alcuni intervistati. Per esempio, Nashawi ha detto (24 maggio 2018):

La competizione di per sé spinge l'artista a fare del suo meglio, e ad essere esposta a più tendenze culturali in una volta sola. Questo è un bene di per sé. Il problema sorge quando l'intero campo artistico e culturale viene mercificato dall'industria della crisi.

Un ostacolo che pare meno aggirabile è la mancanza di status legale in Libano per la gran parte dei profughi siriani (Janmyr 2016). In questo caso, la maggior parte di loro preferisce non palesarsi nello spazio pubblico, o avere in qualche modo esposizione esterna, per timore di venire arrestati o essere deportati in Siria. Questo ha un impatto indubbiamente negativo sulla loro possibilità di diventare artisti pubblici, e di garantirsi una certa sostenibilità economica.

Inoltre, nel caso dei rifugiati in Libano, emerge tra le sfide maggiori la scarsa possiblità di muoversi sia all'interno del territorio libanese che sul piano regionale. La maggior parte degli artisti non gode di ottime condizioni lavorative né di facile mobilità, e l'aver scelto questo percorso professionale complica ulteriormente la loro vita quotidiana. Ad esempio, Rasha Salah della Dar an-Nimer afferma (13 giugno 2018):

Sono semplicemente una consulente qui, questo contratto non mi offre nessuna garanzia. Per lavorare come si deve un artista spesso necessita spostarsi e viaggiare. Per non parlare di quando noi palestinesi vogliamo creare le nostre associazioni... Lo fanno soltanto quelli che sono riusciti ad ottenere la cittadinanza libanese. Altrimenti, dobbiamo beneficiare del supporto di qualche libanese che desidera vedere le nostre attività avviate. Anche il passaporto definisce il tipo di artista che puoi essere.

In questo contesto, l'arte diventa un mezzo terapico per persone vulnerabili socialmente ed economicamente, o uno strumento di attivismo politico utilizzato da locali e profughi. In base alle interviste condotte nel 2018 con esponenti del mondo 
culturale e artistico nel Libano contemporaneo, abbiamo rilevato un processo di mimetizzazione delle tendenze culturali - prevalentemente motivata da propositi politici - con iniziative di sapore umanitario. I finanziamenti, prevalentemente provenienti dall'Unione Europea, dalle agenzie delle Nazioni Unite e da altre organizzazioni non-governative occidentali, a detta delle intervistate, arrivano difatti soltanto se l'ente finanziabile si configura come umanitario, e pertanto inteso a depoliticizzare i soggetti su cui interviene e a rendere la società locale coesa. Difatti, le artiste intervistate hanno spesso affermato di dover celare le proprie posizioni politiche quando negoziano i finanziamenti con alcuni enti internazionali. Nonostante la gran parte degli eventi e iniziative artistiche e culturali abbiano una motivazione politica alla base, quest'ultima non può dunque essere ostentata come tale. Di conseguenza, a detta delle artiste intervistate, l'azione artistica e culturale ampiamente intesa ha l'effetto di "esteticizzare" lo stile di vita locale e parte dello spazio urbano, limitatamente ai distretti che si possono permettere commissioni culturali e artistiche. Ciò che emerge è una versione estetica dell'arte e della produzione culturale, mentre gli artisti, sia libanesi che profughi, continuano a evidenziarne il valore politico: «Penso che il mio lavoro possa aiutare a diffondere idee e ispirare altri a lavorare per il cambiamento», spiega ad esempio Abdul Rahman Katanani, pittore e scultore che utilizza i materiali del quotidiano, piante e fiori per le sue opere, rifugiato palestinese nato a Sabra, nella periferia sud di Beirut, nel 1983.

41 Tania El Khoury, che da anni s'interroga sul legame tra produzione culturale e potenziale politico all'interno della produzione artistica, prova ad andare oltre questo apparente dilemma attraverso il coinvolgimento diretto degli spettatori all'interno delle proprie opere:

L'elemento politico - sottolinea l'artista libanese - non dovrebbe essere percepito come naturale sinonimo di progresso o trasformazione. Storicamente, alcuni artisti sono coinvolti nella sfera politica contribuendo a pratiche di oppressione, foraggiando i regimi che si basano sull'apartheid e l'occupazione militare, ed esercitando il soft power dei governi occidentali nei paesi in via di sviluppo. Inoltre, si è diffuso l'equivoco per cui un'opera d'arte è politica (progressista o meno) se ha un contenuto che si relaziona agli affari odierni. Trovo questa idea semplicistica e scivolosa. Anche se siamo attivisti nella vita quotidiana, mi trovate scettica a presentare la mia pratica artistica solamente in termini di attivismo. Presentare un'opera d'arte come attivismo corre il rischio di silenziare il dibattito e il potenziale politico che esso può presentare. È cruciale per coloro a cui interessa effettuare un cambiamento nella società che il lavoro artistico non rifletta necessariamente la nostra politica individuale. Al contrario, ogni lavoro, con le sue specificità, ha bisogno di inserirsi nella politica della forma, dello spazio e delle comunità che incontra. Sono solita iniziare il mio lavoro con una certa motivazione politica per sfidare la narrazione dello Stato, far emergere le dinamiche di potere, o invitare il mio pubblico a sentirsi collettivamente in lutto per coloro che sono stati uccisi, perseguitati e torturati da regimi politici. Il potenziale politico contestuale di questi eventi emerge dopo che i lavori incontrano il proprio pubblico. Cerco quindi di non infondere nel mio lavoro soltanto la mia personale motivazione politica. Difatti, una performance artistica ha un potenziale politico solo quando incontra il proprio pubblico. È nell'interazione con esso che il lavoro artistico acquisisce il potenziale di catalizzare dinamiche sociali preesistenti, trasformare la politica, sfidare e prendersi cura di una società. Il potenziale politico non risiede pertanto nell'avanzare una certa verità al pubblico attraverso un contenuto prestabilito, ma permette al pubblico di trovare la propria verità soggettiva attraverso la loro percezione del lavoro artistico. 
In ultima analisi, lo spazio artistico emerge quindi come un potenziale terreno politico comune in cui le solidarietà locali si intrecciano a quelle tra profughi. L'umanitarizzazione dei finanziamenti che abbiamo discusso finisce invece per approcciare tale spazio in termini di medicalizzazione delle società ospitanti e profughe, tesa al mantenimento o al ripristino dell'ordine sociale e politico.

\section{Conclusione}

Come ha affermato l'antropologo Renato Rosaldo (1989) a riguardo degli immigrati messicani negli Stati Uniti d'America, vi è una correlazione negativa tra potere e cultura. Coloro che non possiedono potere materiale - come i migranti forzati nel caso del presente articolo - non hanno altra scelta che rivitalizzare un discorso "culturalista" teso al rafforzamento di distinzione identitaria per poter promuovere un cambiamento sociale e politico. Nel contesto socio-culturale libanese, come si è visto, il cambiamento è stretto nella morsa dell'esteticizzazione, in relazione alle crisi d'emergenza e alle ondate di consumismo internazionale - che va di pari passo con lo spirito filantropico, che le crisi suscitano nel Medio Oriente attuale.

Comprendere quali siano le vie più efficaci verso una mobilitazione sociale e politica in Libano, resta un quesito che solo i fautori di cultura stessi possono risolvere. Questi ultimi hanno scelto questa via, sia professionale che personale, proprio in qualità di attivisti. Tuttavia, il loro intento deve sfidare la natura ancor oggi elitaria della cultura in Libano. Anche se, come abbiamo discusso, iniziative come la Dar an-Nimer tentano di portare l'arte e la memoria ai campi profughi invece che chiudersi in spazi inaccessibili, restano iniziative di numero ridotto. I problemi nei trasporti, la priorità di una vita economica in cui la cultura fatica a farsi spazio, e il Beirut-centrismo della vita culturale nel paese, fanno sì che la maggioranza della popolazione locale e migrante in Libano resti estranea a tali forme di resistenza culturale. L'azione resiliente della cultura fatica pertanto a sfidare il regime politico del Libano contemporaneo e il suo ordine geopolitico. Tale azione resiliente è sinonimo stesso di sopravvivenza culturale, specialmente per i gruppi migranti e profughi in Libano.

Le diverse voci locali e migranti a cui abbiamo voluto dedicare ampio spazio all'interno di questo articolo, aprono gli occhi dello spettatore esterno a uno scenario che, da un lato, resta principalmente politico nonostante l'umanitarizzazione dei fondi, ampiamente innestata da numerose agenzie internazionali che offrono risorse a patto di neutralità politica. Dall'altro, è uno scenario troppo spesso definito "attivista", poiché l'arte non sempre porta a trasformazioni socio-politiche progressiste, ma talvolta compiace i regimi politici vigenti o, in ogni caso, viene resa funzionale rispetto al proprio background politico, erigendosi come mero veicolo delle posizioni personali dell'artista.

Infine, se i fondi prevalentemente "umanitari" dovessero riuscire a de-elitizzare la cultura in Libano in tempi di migrazioni e incertezza geopolitica, resterebbe una questione di fondamentale importanza: i fautori della cultura possono mantenere e veicolare il loro messaggio politico originario in tali circostanze? Il successo delle resistenze dipende in gran parte da tali processi individuali e collettivi di opposizione non solo a forze politiche regionali, internazionali o locali, ma anche ai motori stessi della cultura e alla politica economica che la sottende. 


\section{BIBLIOGRAFIA}

Abu Hajar M., 2018 «The Problem with Neutral Songs», in Syria Untold, https://syriauntold.com/ 2018/07/12/the-problem-with-neutral-songs/, 15 novembre 2019.

Baumann H., 2012 «The 'New Contractor Bourgeoisie' in Lebanese Politics: Hariri, Mikati and Fares», in A. Knudsen, Kerr, M. (eds), Lebanon. After the Cedar Revolution, Oxford University Press, Oxford, UK: 125-144.

Das V., Kleinman A., Lock M.M., Ramphele M., Reynolds P. (eds), 2001 Remaking a World. Violence, Social Suffering and Recovery, University of California Press, Oakland, CA.

Das V., Poole D. (eds), 2004 Anthropology in the Margins of the State, School of American Research Press, Santa Fe, NM.

Fassin D., Rechtman, R., 2009 The Empire of Trauma. An Inquiry into the Condition of Victimhood, Princeton University Press, Princeton, NJ.

Haugbolle S., 2012 «History and Memory in Lebanon since 2005. Blind Spots, Emotional Archives and Historiographic Challenges», in A. Knudsen, Kerr, M. (eds), Lebanon. After the Cedar Revolution, Oxford University Press, Oxford, UK: 185-202.

Humphrey M., 2011 «The Special Tribunal for Lebanon: Emergency Law, Trauma and Justice», in Arab Studies Quarterly, 33, vol. 1: 4-22.

Janmyr M., 2016 «The Legal Status of Syrian Refugees in Lebanon», AUB Policy Institute Working Paper.

Khalaf S., 2006 The Heart of Beirut. Reclaiming the Bourj, Saqi Books, London, UK.

Mac Ginty R., 2007 «Reconstructing Post-war Lebanon: A Challenge to the Liberal Peace? », Conflict, Security and Development, 7, vol. 3: 457-482.

Makdisi U., 2000 The Culture of Sectarianism. Community, History, and Violence in Nineteenth-Century Ottoman Lebanon, University of California Press, Oakland, CA.

Mouawad J., Baumann H., 2017 «Wayn al-Dawla? Locating the Lebanese State in Social Theory», Arab Studies Journal, 25, vol. 1: 66-91.

Pahwa S., Winegar J., 2012 «Culture, State and Revolution», in Current History, 112, vol. 758: 338-344.

Rosaldo R., 1989 Culture and Truth: The Remaking of Social Analysis, Routledge, London, UK. Reno W., 2000 «Shadow States and the Political Economy of Civil Wars», in M. Berdal, Malone, D. M., (eds), Greed and Grievance: Economic Agendas in Civil Wars, Lynne Rienner Publishers, London, UK: 43-68.

Scheid K., 2009 «Missing Nike: On Oversights, Double Sights, and Universal Art Understood through Lebanon», in Museum Anthropology, 32, vol. 2: 99-118.

Scheid K., 2015 «Divinely Imprinting Prints, or, How Pictures became Influential Persons in Mandate Lebanon», in C. Schayegh, Arsan, A. (eds), The Routledge Handbook of the History of the Middle East Mandates: 349-369.

Winegar J., 2006 Creative Reckonings: The Politics of Art and Culture in Contemporary Egypt, Stanford University Press, Stanford, CA. 


\section{NOTE}

1. Estella Carpi ha lavorato a questo articolo grazie al supporto offerto dallo European Research Council nel quadro del progetto "Analysing South-South Humanitarian Responses to Displacement from Syria: Views from Lebanon, Jordan and Turkey" (sotto lo European Union's Horizon 2020 Research and Innovation Program, Grant Agreement No. 715582) presso la Migration Research Unit, University College London.

\section{RIASSUNTI}

Sulla base di interviste condotte nel 2018, questo articolo analizza le somiglianze e le differenze che intercorrono tra le sfide che i "fautori della cultura" - artiste in primis - cittadine libanesi e rifugiate palestinesi e siriane devono affrontare nel contesto libanese. Dopo un'illustrazione dello scenario storico-politico libanese e di come in esso la "resistenza culturale" emerge in modo poliedrico, gli autori individuano aree d'incontro e di potenziale solidarietà tra gruppi. L'articolo discute la cosiddetta "umanitarizzazione" dei finanziamenti, attraverso la quale vengono sostenuti e potenziati soprattutto i progetti artistici che possono fungere da strumento di neutralità politica e di "medicalizzazione" dei traumi post-guerra. Tale fenomeno genera in parte una depoliticizzazione ed esteticizzazione dell'arte, "demobilitando" quindi la verve politica dietro al lavoro culturale e, allo stesso tempo, lega la sopravvivenza materiale di tali spazi culturali a cicliche crisi umanitarie.

Based on interviews conducted during 2018, this article examines the challenges that Lebanese citizen, Palestinian and Syrian refugee "culture-makers" - primarily artists - need to face in the Lebanese context, and how such challenges differ from or overlap with one another. After providing an overview of Lebanese political history and how, within it, "cultural resistance" emerges in a multifaceted way, the authors identify areas of encounter and of potential solidarity between groups. The article discusses the so-called "humanitarianization" of funding, through which especially the artistic projects that can serve as instruments of political neutrality and of "medicalization" of post-war traumas are supported. This phenomenon generates in part a depoliticization and aestheticization of art, thus demobilizing the political verve behind cultural work and, at the same time, linking the material survival of such cultural spaces to cyclical humanitarian crises.

\section{INDICE}

Parole chiave : Libano, migrazione forzata, arte, resistenza, cultura

Keywords : Lebanon, forced migration, art, resistance, culture

\section{AUTORI}

\section{ESTELLA CARPI}

Department of Geography, University College London estella.carpi@gmail.com 


\section{STEFANO FOGLIATA}

Dipartimento di Sociologia e Ricerca Sociale, Università degli Studi di Trento stefano.fogliata@unitn.it 